\title{
A Review of Gastric Cancer Research in Malaysia
}

\author{
Kean Ghee Lim*, Kandasami Palayan
}

\begin{abstract}
Incidence rates of gastric cancer in Malaysia has declined by $48 \%$ among males and $31 \%$ among females in the latest reporting period of 13 years. Malays used to have age-standardized-rates only a fifth of those in Chinese and Indians, but the incidence among them is slightly rising even as the rates drop in the other races. Besides ethnicity, a low level of education, high intake of salted fish and vegetables, H pylori infection and smoking are risk factors. Consumption of fresh fruit and vegetable is protective. Variation in the strains of $H$ pylori infection affect gastric cancer risk, with hspEAsia isolates among Chinese appearing linked to a high incidence than with hpAsia2 or hpEurope strains among Indians and Malays. It was reported in the 1980 s that only about $3 \%$ of patients presented with early gastric cancer, but more encouraging rates reaching 27\% with Stage 1 and 2 disease have been reported in the twenty-first century from leading centres. More tumours occur in the distal stomach except in Kelantan, where the incidence is low and main site is the cardia. Prompt endoscopy is advocated and open access, with direct referrals, to such services using a weighted scoring system should be more utilized. In view of the high rate of late disease laparoscopic staging unnecessary laparotomy needs to be avoided. Late presentation of gastric cancer however, is still predominant and the mortality to incidence ratio is relatively high. Besides seeking to reduce risk factors and achieve early detection, implementation of improved care for patients with late disease must be promoted in Malaysia.
\end{abstract}

Keywords: Gastric cancer- Malaysia

Asian Pac J Cancer Prev, 20 (1), 5-11

\section{Introduction}

Malaysia is a country with a moderate risk for gastric cancer (GCa). It remains a major cause of mortality and morbidity although there is evidence it is on the decline. Several hundred Malaysians develop and die from the disease each year (Azizah et al., 2016). Even though we have reports from our National Cancer Registry we do not have a national data base to monitor cancer the patient characteristics and outcomes of our GCa patients. Following the method of similar recent reviews of major disease is Malaysia, a literature search of articles as detailed in the paper Bibliography of clinical research in Malaysia: methods and brief results, using the MESH terms, Stomach Neoplasms, covering the years 2,000 till 2016 was undertaken.(Teng and Zuhanariah, 2014)

69 articles were identified. In brief, clinical research publications containing data on Malaysia for the period Jan 2000 - July 2017 were included (last search date 13 August 2017). Conference proceedings and conference abstracts, relevant theses/dissertation, books/book chapters, reports and clinical practice guidelines were included.

\section{Section 1: Review Of The Literature Epidemiology}

As in the rest of the world, the rates of stomach, or gastric, cancer is declining in Malaysia (Goh, 2007). The latest report of the Penang Cancer registry in the Malaysian National Cancer Registry Report (2007-2011) Azizah et al., (2016) shows the Age Standardized Rate(ASR) for gastric cancer(GCa) in Penang is 6.9 for males and 4.7 for females. This compares to an incidence among males between 1994-1998 (Rokiah M et al., 2003)of 13.3 and for females of 6.8 , a decline of 48 and 31 percent respectively over 13 years.

There are ethnic differences. As with the leading cancers such as breast, colorectal, lung, cervix and nasopharyngeal Chinese have the highest incidence of GCa. Malays had rates 4-5 times lower than Chinese and Indians, but that gaps is closing (Table1) as the incidence among Malays rise and that among the other races decline. Males have higher rates than females (Lim et al., 2008). They had nearly twice the incidence of GCa compared to females in all races but the difference may be decreasing as the overall incidence declines (Azizah et al., 2016). The disease occurs mainly in individuals above the age of 50 years.

In the context of Asia, South-east has a GCa incidence (8.2 for men and 4.1 for women per 100,000 ) less than a third that of East Asia, where the incidence is highest in the world, according to GLOBOCAN 2012 estimates (Ng et al., 2015). The Mortality to Incidence Rate (MIR) for 
$\mathrm{GCa}$ is among the highest for any cancer in South-east Asia (0.88), and holds true for Malaysia.

There are also regional differences within Malaysia (although the National Cancer Registry report of 2007 has reports by states, the data appears unsafe) (Azizah et al., 2016). Malays in Kelantan have been reported to have half the rate of stomach cancer of Malays in Penang. (NorSaleha, 2006) They may have the lowest rates of stomach cancer in the world (Lim, 2009).

\section{Risk Factors}

Goh et al., (2007) conducted a case-control study of 87 cases of GCa (and 174 controls) diagnosed histologically by endoscopy at the University Hospital, Kuala Lumpur. Besides race, the independent risk factors for GCA were; low level of education [odds ratio (OR) 9.8], high intake of salted fish and vegetables (OR 5.2), H. pylori infection (OR 2.5) and smoking (OR 2.5). On the other hand a high intake of fresh fruits and vegetables was an independent protective factor (OR 0.15). As in the rest of the world, the advent of the refrigerator, which has moved the diet of the population away from preserved foods, has probably done more to reduce the incidence of stomach cancer than any other factor. The study was unable to confirm that high chilli intake was protective for GCa. Chilli intake, however, was significant on univariate analysis. Curcumin, an ingredient of turmeric powder used in curries, has been shown to be protective for GCA (Nagabhushan and Bhide, 1992) whereas capsaicin, the chemical that produces the burning sensation, may have a beneficial effect at low dose but the opposite at high doses (Pabalan et al., 2014).

In a study of 50 patients, Karim and Pallesen (2003) found that $10 \%$ were associated with the Ebstein Barr virus, in line with findings elsewhere.

\section{Ethnicity}

Being Chinese had the highest independent risk for GCa (OR 10.23) according to Goh et al., (2007). What the genetic or environmental factors behind this is not clear. Ethnicity has another curious effect in that, although having $\mathrm{H}$. pylori is risk factor for $\mathrm{GCa}$, Indians, who have a high prevalence of $\mathrm{H}$. pylori, appear relatively protected. Among H. pylori positive subjects, only $23.3 \%$ of Indians had GCa, compared to $60.5 \%$ of Chinese and $82.3 \%$ of Malays (Goh et al., 2007). This has also been observed in India and been labelled the "Indian enigma" (Quek and Goh, 2008).

\section{Helicobacter Pylori}

Goh (2009) noted that $43 \%$ of GCa patients had H. pylori between 1989-1990 compared to 37\% between 1999-2000 based on urease test biopsies. Infection rates of $\mathrm{H}$. pylori among all patients endoscoped declined from $52 \%$ to $30 \%$ in the same period. However, the prevalence of H. pylori based on serology among 87 patients with GCa was $79 \%$. It was found to be higher among Indians (82\%) than Chinese (79\%) and Malays. (75\%) (Goh et al., 2007).

In a study of 234 patients with endoscopic antral gastritis in Kelantan, where the prevalence of $\mathrm{H}$. pylori is exceptionally low, Yeh et al., (2009) found histological chronic antral atrophic gastritis in $42.3 \%$, intestinal metaplasia in $7.7 \%$ and $\mathrm{H}$. pylori infection in $6.8 \%$. H. pylori infection was still the only variable factor associated with intestinal metaplasia and dysplasia. They believed this supported the notion 'No H. pylori, no GCa', even though H. pyloriis not the only risk factor necessary.

The low prevalence of $\mathrm{H}$. pylori in Kelantan, may be a key factor for the low incidence of GCa there (Uyub et al., 1994). The diversity of $\mathrm{H}$. pylori strains found in the region, may partially explain the "Indian enigma" (Goh, 2009). Populations with hspEAsia of $\mathrm{H}$. pylori isolates, such as Chinese in Malaysia, exhibit a high incidence of GCa while the incidence is low in populations with a high proportion of hspIndia, hpAsia 2 or hpEurope strains, such as Indians and Malays. (Tay et al., 2009; Breurec et al., 2011; Gunaletchumy et al., 2014)

\section{Clinical Features \\ Staging}

Jasmi et al., (1996a) noted that between 1984 and 1989, only $3.1 \%$ of their GCa patients at HUKM presented with early gastric cancer, but proportion rose to $11.9 \%$ (5.1\% stage 1, 6.8\% stage 2) between 1990-1995. The total number seen had also increased from 97 in the first and 117 in the second period.

Kandasami et al., (2003) on the other hand in a retrospective search of cancers in Ipoh Hospital between 1988-1998 found Stage 1 gastric cancer in only 3.6\% $(9 / 250)$ of their patients, Stage 2 in another 3.6\% (9/25), Stage 3 in $11.2 \%(28 / 250)$ and more than $80 \%(204 / 250)$ had stage 4 disease.

Of the 404 incident cases of GCa in the Penang Cancer Registry between 2004-2008, 182 had the stage of cancer reported. $13.7 \%$ were Stage 1, 13.7\% were stage 2, $31.9 \%$ were stage 3 and $40.7 \%$ were in stage 4 . Caution is warranted regards the accuracy of these percentages as reporting of stage could have been selective.(Azizah et al., 2010) 70 cases of gastric resections with curative intent had surgery in UMMC between 2005 till 2010. 9(13\%) were Stage 1, 10(14\%) in Stage 2, 32(46\%) were Stage 3 and 19 (27\%) were in Stage 4. (Durairaj, 2012)

\section{Pathology \\ Location of tumour}

In Kuala Lumpur, Goh et al., (2007) found that $15(17 \%)$ were proximal, $66 / 87(76 \%)$ of GCas were located distally in the stomach, and 9(7\%) involved the stomach totally. In Penang, Gurjeet (2004) noted that, 9/32 (28\%) gastric carcinomas were located in the cardia or gastro-oesophageal junction while $23 / 32(72 \%$ ) were non-cardia. Durairaj (2012) recorded that $11 / 70(16 \%)$ were proximal, 26/70(37\%) were middle and 33/70(47\%) were distally located in the stomach in his series of resected GCa between 2005 till 2010.

In an earlier series from 1988 to 1998, Kandasami et al., (2003) in Ipoh, found that in 155/250 (62\%) of the patients the cancer was located at the gastric antrum, $23 / 250(9 \%)$ involved the body and antrum, $33 / 250(13 \%)$ involved the body, and in 39/250 (15\%) of the patients, the disease located at the cardia and fundus of the stomach. The predominance of distal tumours appears to correlate 
to an earlier series reported in 1976 from the University Hospital where $53 \%$ of GCa was located in the antrum, $28 \%$ in the body, $17 \%$ in the cardia and fundus and $3 \%$ involved the entire stomach (Bahari et al., 1976).

On the other hand, in sharp contrast in Kelantan 14/23 (61\%) were cardia-located tumours.(Gurjeet et al., 2004).

\section{Histology}

Most GCa are adenocarcinomas. Kalavathy (1993) retrieved and studied gastrectomy specimen form 79 cases in UMMC between 1986 and 1991. 42(53\%) were the intestinal type by Laurén's classification and 37(47\%) were diffuse type with no other type seen. There no age no gender difference in the distribution. In Goh et al.,'s series (2007), 59(68\%) belonged to the intestinal type, according to Laurén's classification, and 28(32\%) were the diffuse type. Similarly, in Penang 23(72\%) were intestinal type, $8(25 \%)$ diffuse and 1(3\%) mixed type. The ratio was also similar in Kelantan with $16(70 \%), 5(22 \%)$ and $29 \%$ ) cases respectively.(Gurjeet et al., 2004) There does not appear to be any evidence $H$ pylori infection is linked to the intestinal type of GCa. Durairaj (2012), on the other hand found $31 / 70(44 \%)$ of a series who had surgery with curative intent had intestinal type, compared to $39 / 70(56 \%)$ with diffuse type and noted a better survival for those with intestinal type.

Durairaj (2012) did not find Bormann's grading significantly associated with survival.

Jasmi et al., (1996b) have reported a series of 7 cases of leiomyosarcoma of the stomach at UKM between 1989 and 1995. All but one were males. The mean age was 56 years Three were Malays. 3 Chinese and I Indian. The tumours were large and exophitic ranging in size from $8-20 \mathrm{~cm}$. Two had liver metastases wrong diagnosed as originating from the pancreas. Three patients were alive after surgery ranging from 6 months to 6 years.

Inflammatory myofibroblastictumour (IMT) is a rare cancer of the stomach, which has been reported in Malaysia in a boy of 13 years. Local recurrence was detected 3 years later with invasion into the pancreas and hilum of the spleen. Wide resection of the stomach, distal pancreatectomy and splenectomy achieved recurrence-free for two years (Hamidah et al., 2007). Gastrointestinal stromal tumours (GIST) are derived from the gastrointestinal mesenchyma, in the gastrointestinal tract and can also occur in the stomach. Siam and Siow have described a 29-year-old Iban man with GIST in the stomach who presented with intussusception (Siam and Siow, 2008). More than half (53\%) of GIST tumours have been noted in the stomach in a Malaysian report (Kkrishnappa et al., 2016)

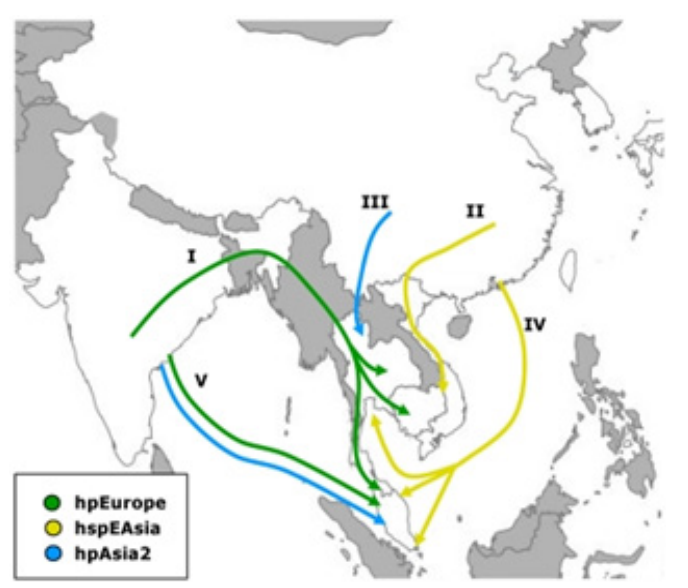

Figure 1. Human Migration into South-East Asia According to $H$. pylori Strain Evidence.(from Breurec et al., 2011)

\section{Biomarkers}

Cheah and Ramachandran (1994) noted that GCa cells from patients in Kuala Lumpur $(\mathrm{n}=79)$ produced more sulphomucins compared to normal controls.

GCa cells, like other cells, exhibit cluster of differentiation (CD) antigens which characterize the response of the immune system to these cells. H pylori infection has been shown to induce the expression of certain CD antigens. Sukri et.al. (2016) have found that CD 5, CD40, CD 71, and CD 39 (antigens perpetuating tolerance of immune cells) were upregulated more than twofold in GCa cells from six patient in HUKM compared to normal mucosa, while CD279 (antigen essential in T cell apoptosis) was upregulated in normal gastric mucosa cells taken from these patients at least $10 \mathrm{~cm}$ from the tumour site.

Methylation of the Deleted in Colorectal Cancer (DCC) gene has been observed in the course of gastric carcinogenesis and disappeared in advanced gastric carcinoma (Hibi et al., 2010). The rs 10502974 SNP which was located within the intronic region of DCC gene was the single nucleotide polymorphism(SNP) most significantly associated with $\mathrm{H}$. pylori infection $(\mathrm{p}=0.00549)$ among Malays in Kelantan (Maran et al., 2013a).

The XRCC1gene, mapped to human chromosome $19 \mathrm{q} 13.2-13.3$, is recognized as a gene related to DNA repair. Three common SNPs, (Arg194Trp), (Arg280His) and (Arg399Gln) are associated with gastrointestinal cancer risk. Halim et.al. noted an increased risk for GCA among 250 patients in Sabah, with OR of 1.89 for those with the (Arg194Trp) SNP and OR of 2.94 for the(Arg399Gln) SNP. The frequency of 194Trp allele was higher than reported in Egypt and Mexico but was lower than those reported in Eastern Asia including Han Chinese

Table 1. Age Standardised Incidence of Gastric Cancer (GCa) in Penang among Various Ethnic Groups

\begin{tabular}{ccccccc}
\hline & & Males & & \multicolumn{2}{c}{ Females } \\
& Malays & Chinese & Indians & Malays & Chinese & Indians \\
\hline $1994-1998$ & 3.0 & 17.7 & 20.3 & 2.2 & 8.5 & 11.5 \\
$1999-2003$ & 3.2 & 12.3 & 9.4 & 1.8 & 7.8 & 10.8 \\
$2004-2008$ & 4.1 & 10.4 & 7.5 & 2.0 & 6.4 & 7.0 \\
\hline
\end{tabular}


gastrointestinal cancer populations. The 399Gln allele frequency was found higher when compared to Egyptian, Han Chinese, Japanese, Korean and Western Mexican populations but was lower than in the Thai population gastrointestinal cancer population.(Halim et al., 2016)

Rajadurai (2017) has found human epidermal growth factor receptor2 (HER2) overexpression, a biomarker of breast cancer, in advanced GCa and gastroesophageal junction cancer. HER2 overexpression was evident in nearly $24.6 \%$ (56/288) of the Malaysian,and significantly more among males and diffuse-type tumors.

\section{In $H$ pylori related $G C A$}

\section{Factors in the bacteria}

By comparing theH. pylori genome of 21 Malaysian and 6 other Asian H. pylori strains, Gunaletchumy et al. (2014) found the presence of outer membrane protein GC26_66 (type 1), hypothetical ATPase protein GC26_69, hypothetical protein GC26_73 and hypothetical protein GC26_33 were shown to be risk factors for gastric cancer development. In addition, outer membrane GC26_77 may have a protective effect against peptic ulcer disease.

$\mathrm{Cag} \mathrm{A}+\mathrm{H}$. pylori infection is known to have a propensity of developing GCa. The CagA protein possesses EPIYA motifs which are key to the function of the protein in phenotypic changes of the bacteria. The EPIYA motifs have been divided into four classes, designated EPIYA -A to -D. Schmidt et al., (2009) founds genes encoding EPIYA-A in $97.6 \%$ and EPIYA-B in $100 \%$ of $\mathrm{H}$. pylori isolated from patients in UMMC, Kuala Lumpur and Changi, Singapore. However, 93.0\% of isolates from Indian patients with functional dyspepsia encoded EPIYA-C while $91.8 \%$ of such Chinese patients encoded EPIPYA-D. $61.5 \%$ of Malay patients with functional dyspepsia and $\mathrm{H}$. pylori infection had isolates with EPIYA-C and 38.5\% EPIYA-D. Only H. pylori isolates from distal GCa patients who were Chinese were studied and $81.7 \%$ of them encoded for EPIYA-D. These findings may explain the "Indian Enigma".

\section{Factors in the patient}

Schmidt et al., (2011) studied gene polymorphisms(SNPs) related to immune and cancer-related pathways for association with $H$ pylori infection in 422 patients undergoing endoscopy in Malaysia (UMMC) and Singapore and found significant association among Chinese, between SNPs (i) PTGS2-1195G (rs689466) and an increased risk of $\mathrm{GCa}$ on adjusting for $\mathrm{H}$. pylori status (OR 1.53, and (ii) IL1B-1473C (rs1143623) and a decreased risk of GCa (OR 0.64). (iii) EBBR2+1963G (rs1801200) was associated with low H. pylori infection (OR 0.48).

Chronic inflammation of the gastric mucosa, a consequence of $H$ pylori infection, is a risk factor for GCa. A number of nucleotide-binding oligomerization domain(NOD)-like receptors (NLRs) are involved in the inflammatory pathway. Castano-Rodriguez et al., (2014a) investigated the role of genetic polymorphisms and expression of genes involved in the NLR signaling pathway in $\mathrm{H}$. pylori infection and GCa among 310 ethnic Chinese in Malaysia and Singapore (87 non-cardia
GCA cases and 223 controls with functional dyspepsia). CARD8 (rs11672725), NLRP3 (rs10754558), NLRP3 (rs4612666), NLRP (12-rs199475867) and NLRX1 (rs10790286) showed significant associations with GCa. On multivariate analysis, only CARD8 (rs11672725) remained a risk factor (OR 4.80).

Autophagy plays a pivotal role in $H$ pylori infection and inflammation. Defects in autophagy may lead to $H$ pylori-related GCa. In the same cohort of Chinese studied by Castano-Rodriguez et al., (2015) ATG16L1 (rs2241880) increased the risk of GCa (OR 2.38) and H pylori infection (OR: 1.49) while IRGM (rs4958847) decreased GCa risk (OR 0.26).

Toll-like receptors (TLRs), an important part of the innate immune system, may also play a role in $H$ pylori-related GCa. Also studied in that cohort, after multivariate analysis, TLR4 (rs11536889) remained a risk factor for GCa (OR: 3.58). Six other polymorphisms [TLR2 (rs3804100), TLR2 (-196 to $-174 \mathrm{del})$, MD-2 (rs11465996), MD- (rs16938755), LBP (rs2232578) and TIRAP (rs7932766)] increased the risk of GCa in combination with $H$ pylori infection in these Chinese individuals. On the other hand TLR4 (rs10759932) decreased the risk of $H$ pylori infection (OR: 0.59). (Castaño-Rodríguez et al., 2014b). Ram et al., (2015) have found that TLR1 (rs4833095) and TRL-10 (rs10004195) linked with increased levels of leptin, leptin receptor, pro-inflamatory cytokines and low levels of ghrelin associated with Hpylori infection, were also associated with GCa.

Among Kelantan Malays with H. pylori infection and gastric precancerous lesions identified during endoscopy, SNPs rs9315542 (UFM1 gene), rs6878265 (THBS4 gene), rs1042194 (CYP2C19 gene) and rs10505799 (MGST1 gene) were significantly associated with atrophic gastritis, complete intestinal metaplasia, incomplete metaplasia with foci of dysplasia and dysplasia, respectively. There was a higher chance of a subject having the allele compared to controls for rs9315542(0.4 vs 0.06), rs6878265 (0.6 vs 0.01), rs 1042194 (0.6 vs 0.01) and rs10505799 (0.5 vs 0.02).(Maran et al., 2013b)

\section{Management \\ Diagnosis}

Because indications for upper gastrointestinal endoscopy varies, the rate GCa is detected can vary, from $1.4 \%(16 / 1119)$ for patients endoscopy for dyspepsia (Kudva and Thein-Htut, 1988), to 3.4\% (7/208) at a new endoscopy service (Paramarajah, 1985). Goh et al., (2009) reported a rate of $2.8 \%$ and $2.0 \%$ from the same hospital between 1989-1990 and 1999-2000 respectively.

Prompt endoscopy has been advocated for the earlier diagnosis of gastric cancer in East Asia (Chen et al., 2015).

However, it has been argued that the prompt endoscopy advocated for East Asia where the incidence is much higher, may not be applicable to Malaysia (Mahadeva and Goh, 2015). Mahadeva et al., (2008) have proposed that testing for $H$ pylori and treating would be a more cost-effective strategy for Malaysia. Besides the Urea Breath Test and the biopsy urease test, a stool antigen test has been found to be highly sensitive and specific. 
(Osman et al., 2014)

However, in order to facilitate early diagnosis, Tata et al., (2014) have devised, an open access endoscopy service using a weighted scoring system consisting of demographic data (age, race and sex) as well as symptoms (melena, anemia, vomiting, weight loss, epigastric mass/ fullness, dysphagia, early satiety and dyspepsia).

\section{Presentation}

Unfortunately GCa cause no specific symptoms especially in the early stage. Loss of weight and appetite, early satiety, anaemia and a palpable mass appear too late.

Kandasami et al., (2003) found $82 \%$ of their patients were found to have Stage $4 \mathrm{GCa}$, not surprisingly curative surgery wasperformed to only $16 \%$ of the patients. An alarming $33.2 \%$ of the patients did not even receive palliative surgery. At the initial assessment or at time of surgerythe disease was too advanced for any form of surgicalprocedures. In 18 patients $(7.2 \%)$ the abdomen was opened and closed at surgery and in another 109(43.6\%) some palliative procedure was done.

Reviewing 86 patients with stomach cancer in Seremban between 2004 and 2008, Tata et al., (2013) found that the mean duration from the first appearance of cancer symptoms to diagnosis at endoscopy was 32.4 weeks. The main reasons for the long delay were self-medication, empirical treatment for dyspepsia and delay in high risk patients obtaining an appointment for an endoscope procedure.

In unusual cases, GCa has presented in a 27-year old woman with perforation of the stomach at 16 weeks of gestation in her third pregnancy (Jasmi et al., 2000) and with pulmonary lymphangitic carcinomatosis in three women, two of whom were young (Liam et al., 1989). Inferior vena cava obstruction has also been reported as the presenting feature in a 56-year old woman in Malaysia with stage $4 \mathrm{GCa}$ (Patel, 2015).

\section{Assessment}

Treatment strategy in GCa depends heavily on the stage of disease and staging assessment is mandatory.

In the context of the low rate of resectable stomach cancers in Malaysia (Kandasami et al., 2003), Mahadevan et al., (2010) have evaluated the role of laparoscopic staging in an effort to reduce unwarranted open laparotomy in patients with GCA. Computed tomography (CT) scan often under-stage disease and endoscopic ultrasound is not widely available in Malaysia. After excluding patients with obvious metastatic disease, they enrolled 40 patients for laparoscopic staging. Laparoscopic staging detected $90.3 \%$ of T3 tumours compared with $58 \%$ detected by CT. On the other hand the sensitivity of laparoscopic staging in detecting nodal disease $(70 \%)$ was not much higher than CT (62.5\%). Laparoscopy detected peritoneal metastases in 7 out of the 40 cases $(17.5 \%)$ none of which were noted on CT scan, avoiding unnecessary laparotomy in these patients (Kandasami, 2012). Siow et al., (2017) have investigated and advocated the placement of a T-tube feeding jejunostomy at the same laparoscopy staging procedure.

\section{Outcome}

In his series 70 patients with GCa undergoing resection with curative intent, Durairaj (2012) noted the mean size of tumour was $59.6 \mathrm{~mm}$ and $28.6 \%$ had surgical margins involved. No patients underwent neoadjuvant therapy but $24(34 \%)$ had adjuvant chemotherapy including 4 with concurrent radiotherapy. There were 16 patients with post-operative complications and 5 post-operative deaths. The overall survival was $71 \%$ at 1 year and $46 \%$ at 3 years. Tumour size and location, Lauren's intestinal type and differentiation of tumour, surgical margins, lymphovascular invasion and stage of cancer were the tumour factors associated with survival.

\section{Section 2: Relevance of Findings for clinical practice}

It is heartening to note that the burden of GCA is declining. Nevertheless, it still remains a major cause of morbidity and mortality in Malaysia. With its high Mortality to Incidence rate, several hundred Malaysians are estimated to die from GCA each year. The effort that needs to be made to improved survival for those who will get the disease is to achieve early detection. In this regard, open access upper GI endoscopy among those identified with high risk using a scoring system should be more widely applied, especially in areas where the incidence of GCA is appears to be higher, such as the states of Sabah, Sarawak, Penang, Johore and Negeri Sembilan.

The eradication of $H$. pylori, avoidance of smoking, pickled vegetables and salted/smoked foods which have already been responsible for a declining incidence of GCA should not be overlooked.

\section{Section 3: Future Research Direction}

We have to take note and manage the reality that most of our patients will continue to present with late disease. In this regard determining the most appropriate chemotherapy with or without radiotherapy in the face of our resources is most appropriate for palliation should be investigated. Feeding regimes, supportive care and other ancillary services that best meet the needs of patients in their different settings need to be determined. As treatment strategies become more specific to disease stage, the training of surgical and supportive staff to meet such needs should also be addressed. How best to position tertiary hospitals to cater for our population in Malaysia also warrants study.

\section{Acknowledgements}

We wish to thank Prof Teng Cheong Lieng for once again doing the literature search.

\section{References}

Azizah AM, Devaraj T, Saraswati BR (2010) Penang Cancer Registry Report No.9 2004-2008. Penang State Health Department.

Azizah AM, Nor Saleha IT, Noor Hashimah A, et al (Eds) (2016). Malaysian National Cancer Registry Report 2007-2011.

Bahari HM, Ti TK, Yong NK (1976). Gastric carcinoma - a 
review of 114 cases. Med J Malaysia, 31, 120-2

Breurec S, Guillard B, Hem S, et al (2011). Evolutionary history of Helicobacter pylori sequences reflect past human migrations in Southeast Asia. PLoS One, 6, e98899.

Castaño-Rodríguez N, Kaakoush NO, Goh K-L, Fock KM, Mitchell HM (2014a). The NOD-like receptor signalling pathway in Helicobacter pylori infection and related gastric cancer: A case-control study and gene expression analyses. PLoS One, 9, e22058.

Castaño-Rodríguez N, Kaakoush NO, Pardo AL, et al (2014b). Genetic polymorphisms in the Toll-like receptor signalling pathway in Helicobacter pylori infection and related gastric cancer. Hum.Immunol, 75, 808-15.

Castaño-Rodríguez N, Kaakoush NO, Goh K-L, Fock KM, Mitchell HM (2015). Autophagy in Helicobacter pylori infection and related gastric cancer. Helicobacter, 20, 353-69.

Cheah Pl, Ramachandran K (1994). Alterations in mucin type: an indicator for suspicion of malignant gastric transformation. Malays J Pathol, 16, 39-42.

Chen SL, Gwee KA, Lee JS, et al (2015). Systematic review with meta-analysis: prompt endoscopy as the initial management strategy for uninvestigated dyspepsia in Asia. Aliment Pharmacol Ther, 41, 239-52.

Durairaj GG (2012) Surgical outcomes of gastric resections with curative intent for adenocarcinoma of the stomach: a University Malaya Medical Centre(UMMC) experience. Master of Surgery Dissertation, WO100 UM 2012 Gun.

Goh KL (2007). Changing trends in gastrointestinal disease in the Asia-Pacific region. J Dig Dis, 8, 179-85.

Goh KL (2009). Epidemiology of Helicobacter pylori infection in Malaysia-observations in a multiracial Asian population. Med J Malaysia, 64, 187-92.

Goh K-L, Cheah P-L, Md N, Quek K-F, Parasakthi N (2007). Ethnicity and $\mathrm{H}$. Pylori as risk factors for gastric cancer in Malaysia: A prospective case control study. Am J Gastroenterol, 102, 40-5.

Goh K-L, Wong H-T, Lim C-H, Rosaida MS (2009). Time trends in peptic ulcer, erosive reflux oesophagitis, gastric and oesophageal cancers in a multiracial Asian population. Aliment. Pharmacol Ther, 29, 774-80.

Gunaletchumy SP, Seevasant I, Tan MH, et al (2014). Helicobacter pylori genetic diversity and gastro-duodenal diseases in Malaysia. Sci Rep, 4, 7431.

Gurjeet K, Subathra S, Bhupinder S (2004). Differences in the pattern of gastric carcinoma between north-eastern and north-western peninsular Malaysia: a reflection of Helicobacter pylori prevalence. Med J Malaysia, 59, 560-1.

Halim NHA, Chong ETJ, Goh LPW, et al (2016). Variant alleles in XRCC1 Arg194Trp and Arg399Gln polymorphisms increase risk of gastrointestinal cancer in Sabah, North Borneo. Asian Pac J Cancer Prev, 17, 1925-31.

Hamidah A, Khu SY, Thambidorai CR, et al (2007). Recurrent gastric inflammatory myofibroblastic tumour in a 13-year-old male. Pediat Surg Int, 23, 601-3.

Hibi K, Sakata M, Sakuraba K, et al (2010). Methylation of the DCC gene is lost in advanced gastric cancer. Anticancer Res, 30, 107-9.

Jasmi AY, Haron A, Noraishah MA, et al (1996a). A 12 year review of gastric cancer at the Universiti Kebangsaan Malaysia : Is the trend Changing?. J Gastroenterol Hepatol, 11, 2:A53.

Jasmi AY, Haron A, Swaminathan S, Hayati AR, Isa Rose (1996b). Leiomyosarcoma of the Stomach: A Clinicopathological Review. J Gastroenterol Hepatol, 11, 2:A52.

Jasmi AY, Normala B, al-Amin MD (2000). Perforated malignant gastric ulcer in a pregnant young adult: a case report. Med
J Malaysia, 55, 135-7.

Kalavathy (1993). Mucin patterns of intestinal and diffuse types of gastric carcinoma in Malaysians. Master of Pathology Dissertation, University of Malaya, QZ365 Kal

Kandasami P, Tan WJ, Norain K (2003). Gastric cancer in Malaysia: the need for early diagnosis. Med J Malaysia, 58, 758-62.

Kandasami P (2012). The efficacy of pre-operative laparoscopy in the staging for gastric cancer. IeJSME, 6, 103-5.

Karim N, Pallesen G (2003). Epstein-Barr virus (EBV) and gastric carcinoma in Malaysian patients. Malays J Pathol, 25, 45-7.

Kkrishnappa P, Loh EJ, Mohamad IB, et al (2016). Histomorphology and immunohistochemistry of gastrointestinal stromal tumors in a Malaysian population. Asian Pac J Cancer Prev, 17, 2795-9.

Kudva MV, Thein-Htut N (1988). Upper gastrointestinal endoscopy as an initial investigation in dyspepsia--a Malaysian experience. Med J Malaysia, 43, 311-7.

Liam CK, Looi LM, Pailoor J, Alhady SF (1989). Dyspnoea in young adults due to pulmonary lymphangitic carcinomatosis from gastric carcinoma. Ann Acad Med, 18, 713-6.

Lim GCC, Rampal S, Halimah Y(Eds) (2008). Cancer incidence in Peninsular Malaysia, 2003-2005. National Cancer Registry. Kuala Lumpur.

Lim KG (2009). Malays in peninsular Malaysia may have the lowest incidence of stomach cancer in the world. Med $J$ Malaysia, 64, 91-2.

Mahadeva S, Goh K-L (2015). Letter: prompt endoscopy in Asians with uninvestigated dyspepsia. Aliment Pharmacol Ther, 41, 792.

Mahadeva S, Chia Y-C, Vinothini A, Mohazmi M, Goh K-L (2008). Cost-effectiveness of and satisfaction with a Helicobacter pylori "test and treat" strategy compared with prompt endoscopy in young Asians with dyspepsia. Gut, 57, 1214-20.

Mahadevan D, Sudirman A, Kandasami P, Ramesh G (2010). Laparoscopic staging in gastric cancer: An essential step in its management. J Minimal Access Surg, 6, 111-3.

Maran S, Lee YY, Xu S, et al (2013a). Deleted in colorectal cancer (DCC) gene polymorphism is associated with H. pylori infection among susceptible Malays from the north-eastern region of Peninsular Malaysia. Hepatogastroenterol, 60, 124-8.

Maran S, Lee YY, Xu S, et al (2013b). Gastric precancerous lesions are associated with gene variants in Helicobacter pylori-susceptible ethnic Malays. World J Gastroenterol, 19, 3615-22.

Nagabhushan M, Bhide SV (1992). Curcumin as an inhibitor of cancer. J Am Coll Nutr, 11, 192-8.

Ng CJ, Teo CH, Abdullah N, Tan WP, Tan HM (2015). Relationships between cancer pattern, country income and geographical region in Asia. BMC Cancer, 15, 613.

NorSaleha IT (2006). Kelantan Cancer Registry Report 1999-2003.

Osman HA, Hasan H, Suppian R, Bahar N, Hussin NS (2014). Evaluation of the atlas Helicobacter pylori stool antigen test for diagnosis of infection in adult patients. Asian Pac J Cancer Prev, 15, 5245-7.

Pabalan N, Jarjanazi H, Ozcelik H (2014). The impact of capsaicin intake on risk of developing gastric cancers: a meta-analysis. $J$ Gastrointest Cancer, 45, 334-41.

Paramarajah S (1985). Experience with gastroscopy in the diagnosis of stomach cancer in Malaysia. Southeast Asian J Trop Med Public Health, 16, 609-12.

Patel SA (2015). The inferior vena cava (IVC) syndrome as the initial manifestation of newly diagnosed gastric 
adenocarcinoma: a case report. J Med Case Rep, 9, 204.

Quek KF, Go, K-L (2008). Observations of the Indian Enigma is valid. Am J Gastroenterol, 103, 1575.

Rajadurai P, Fatt HK, Ching FY (2017). Prevalence of HER2 positivity and its clinicopathological correlation in locally advanced/metastatic gastric cancer patients in Malaysia. J Gastrointest Cancer, doi: 10.1007/s12029-017-9921-1. [Epub ahead of print].

Ravishankar Ram M, Goh KL, Leow AHR, et al (2015). Polymorphisms at Locus 4p14 of Toll-Like Receptors TLR-1 and TLR-10 confer susceptibility to gastric carcinoma in Helicobacter pylori infection. PLoS One, 10, e0141865.

Rokiah M, Devaraj T, Zahirah MZ, et al (2003). Penang cancer registry report 1994-1998. Penang State Health Department.

Schmidt H-MA, Goh K-L, Fock KM, et al (2009). Distinct cagA EPIYA motifs are associated with ethnic diversity in Malaysia and Singapore. Helicobacter, 14, 256-63.

Schmidt H-MA, Ha DM, Taylor EF, et al (2011). Variation in human genetic polymorphisms, their association with Helicobacter pylori acquisition and gastric cancer in a multi-ethnic country: SNPs in H. pylori-related disease. J Gastroenterol Hepatol, 26, 1725-32.

Siam FA, Siow SL (2008). Stomach gastrointestinal stromal tumours (GIST) intussuscepted into duodenum: A case report. Malays J Med Sci, 15, 68-70.

Siow SL, Mahendran HA, Wong CM, Milaksh NK, Nyunt M (2017). Laparoscopic T-tube feeding jejunostomy as an adjunct to staging laparoscopy for upper gastrointestinal malignancies: the technique and review of outcomes. $B M C$ Surg, 17, 25.

Sukri A, Hanafiah A, Kosai NR, et al (2016). Surface antigen profiling of Helicobacter pylori-infected and -uninfected gastric cancer cells using antibody microarray. Helicobacter, 21, 417-27.

Tata MD, Dharmendran R, Ramesh G, Kandasami P (2013). Delay in diagnosis of upper gastrointestinal cancer: whose fault is it?. Med J Malaysia, 68, 275-7.

Tata MD, Gurunathan R, Palayan K (2014). MARK's Quadrant scoring system: a symptom-based targeted screening tool for gastric cancer. Ann Gastroenterol, 27, 34-41.

Tay CY, Mitchell H, Dong Q, et al (2009). Population structure of Helicobacter pylori among ethnic groups in Malaysia: recent acquisition of the bacterium by the Malay population. BMC Microbiol, 9, 126.

Teng CL, Zuhanariah MN, Ng CS, et al.(2014). Bibliography of clinical research in Malaysia: methods and brief results. Med J Malaysia, 69, 4-7.

Uyub AM, Raj SM, Visvanathan R, et al (1994). Helicobacter pylori infection in north-eastern peninsular Malaysia. Evidence for an unusually low prevalence. Scand $J$ Gastroenterol, 29, 209-13.

Yeh LY, Raj M, Hassan S, et al (2009). Chronic atrophic antral gastritis and risk of metaplasia and dysplasia in an area with low prevalence of Helicobacter pylori. Indian $J$ Gastroenterol, 28, 49-52.

This work is licensed under a Creative Commons AttributionNon Commercial 4.0 International License. 